\title{
IS CIRCULATING GLA-RICH PROTEIN LINKED WITH CORONARY CALCIUM AND CARDIOVASCULAR PATHOLOGY IN PATIENTS WITH ATRIAL FIBRILLATION OR HEART FAILURE? A PILOT STUDY
}

\author{
Deyana Vankova ${ }^{1}$, Milena Pasheva ${ }^{1}$, Atanas Angelov ${ }^{2}$, Yoto Yotov ${ }^{2}$, Bistra Galunska ${ }^{1}$ \\ ${ }^{1}$ Department of Biochemistry, Molecular Medicine and Nutrigenomics, \\ Faculty of Pharmacy, Medical University of Varna \\ ${ }^{2}$ First Department of Internal Diseases, Faculty of Medicine, Medical University of Varna
}

\begin{abstract}
INTRODUCTION: Nowadays Gla-rich protein (GRP) is recognized as a novel biomarker playing a pivotal role in the crosstalk between chronic inflammation and vascular calcification.

AIM: The aim of this article is to study the link between circulating GRP, cardiovascular pathology, and the degree of arterial calcification evaluated by the coronary arterial calcium score (CACS) in a Bulgarian population sample.

MATERIALS AND METHODS: Adult participants $(n=81)$ of both genders were divided into: controls $(n=41)$ - subjects with estimated moderate-to-high risk without known cardiovascular diseases (CVDs) and a combined CVD group $(n=40)$ - patients with paroxysmal or persistent atrial fibrillation in sinus rhythm, and heart failure subjects with preserved ejection fraction. A structured interview was carried out for evaluation of the classical CVD risk factors. CACS was determined by multislice computed tomography. Routine laboratory parameters were extracted from medical records. Serum levels of total GRP, matrix Gla protein, and osteocalcin were estimated by commercial ELISA kits. Standard statistical methods (descriptive statistics, Student's t-test and Spearman's correlation) were applied. Statistical significance was considered at $\mathbf{p}<0.05$.
\end{abstract}

RESULTS: Significantly lower GRP levels were established in patients with coronary calcium compared to those without calcium deposits. Clear tendency for decreased levels of GRP was observed in the combined CVD group vs controls. Circulating GRP significantly correlates with uncarboxylated matrix Gla protein. An association between serum GRP, CRP, and low-density lipoproteins (LDLs) was demonstrated.

CONCLUSION: This study adds new information regarding the role of circulating GRP as a new player in calcification inhibition. Our findings illuminate the link between total circulating GRP, CVD pathology, and the degree of coronary calcification.

Address for correspondence:

Deyana Vankova

Faculty of Pharmacy

Medical University of Varna

84 Tzar Osvoboditel Blvd

9002 Varna

e-mail: deyana.vankova@mu-varna.bg

Received: February 19, 2021

Accepted: March 15, 2021
Keywords: Gla-rich protein (GRP), coronary arterial calcium score (CACS), atrial fibrillation, heart failure 


\section{INTRODUCTION}

As cardiovascular diseases (CVDs) are one of the major causes of death worldwide, a significant part of scientific research is focused on elucidating the molecular mechanisms underlying their development and progression. Vascular calcification (VC) is a process of calcium phosphate deposition in the vessel wall due to impaired balance between the regulatory mechanisms that contribute to the mineralization of the vessel wall and those that inhibit it (1). VC is a multifactorial process, but the loss of calcification inhibitors is a major mechanism for its enhancement $(2,3)$.

Gla-rich protein (GRP) is a circulating vitamin $\mathrm{K}$-dependent protein, also known as an upper zone of growth plate and cartilage matrix associated protein (UCMA) (4). A decade earlier GRP has been suggested to act as a negative regulator of osteogenic differentiation, a novel secretory cartilage-specific protein with implications in osteogenesis, a modulator of calcium availability in the extracellular matrix (ECM), and as a potential inhibitor of soft tissue calcification $(4,5)$. In addition, it plays a role as an inhibitor of pathological VC and as an anti-inflammatory agent in the cardiovascular system $(6,7)$. Nowadays GRP is recognized as a novel biomarker, which plays a pivotal role in the crosstalk between chronic inflammation and calcification in the articular, renal and cardiovascular systems (8).

Similarly to matrix Gla protein (MGP), another vitamin $\mathrm{K}$-dependent protein, acting as local inhibitor of soft tissue calcification and vascular mineralization $(9,10)$, GRP $\gamma$-carboxylation is essential for its calcification inhibitory function (8). In the study of Viegas et al. (6) it was demonstrated that GRP expression and accumulation are upregulated by a calcification process and possibly GRP is a component of the fetuin-A-MGP calcification inhibitory system. Moreover, it was demonstrated that GRP is included in the fetuin-A-MGP complex at the sites of valvular calcification. Also, only the $\gamma$-carboxylated GRP inhibits effectively calcification and osteochondrogenic differentiation through $\alpha$-smooth muscle actin upregulation and osteopontin downregulation. In the same in vitro study it was demonstrated that addition of carboxylated GRP is able to reduce both vascular calcification and proinflammatory responses of articular and immune cells $(6,8)$. GRP calciumbinding properties and its association to calcification processes indicate that its function might be associated with prevention of calcium-induced signaling pathways and direct mineral binding leading to blockage of crystal formation and maturation. High levels of GRP are found in sites of pathological calcification possibly due to its capability to bind calcium phosphate crystals directly (11). An impaired $\gamma$-carboxylation status of GRP is considered to be associated with pathological calcification, including vascular calcification, osteoarthritis, and certain cancers $(11,12)$. It is considered that circulating GRP is a systemic inhibitor of ectopic calcification through its involvement in the inhibition of $\mathrm{Ca} / \mathrm{P}$ mineral crystal formation and maturation, with profound consequences on vascular calcification (7).

The studies examining the role of circulating GRP as a novel marker of vascular calcification and inhibitor of inflammation are extremely scarce. To our knowledge, circulating GRP levels and their link to CVD have not examined in Bulgarian population.

\section{AIM}

The aim of our pilot study was to examine the link between circulating GRP, CVD pathology and the degree of arterial calcification evaluated by the CAC score in Bulgarian population sample.

\section{MATERIALS AND METHODS}

\section{Patients and Methods}

The current study included 81 Bulgarian patients who were admitted to St. Marina University Hospital Varna between October 2018 and January 2020. In the control group were included 41 subjects who were estimated to be with moderate-to-high risk for CVD but without known CVD. The other 40 subjects formed the combined CVD group-23 patients with paroxysmal and persistent atrial fibrillation (AF) and 17 patients with heart failure (HF) with preserved ejection fraction (ejection fraction $>40 \%$, HFpEF) in sinus rhythm at the time of hospitalization. Information about age and history of CVD, smoking status, presence and treatment of arterial hypertension, hyperlipidemia, type 2 diabetes mellitus, and current use of anticoagulant medication were collected through a structured interview. Hypertension was defined as blood pressure 
Deyana Vankova, Milena Pasheva, Atanas Angelov et al.

(BP) $>140 / 90 \mathrm{mmHg}$ at the time of examination or with a history of elevated $\mathrm{BP}$ and on antihypertensive medication. According to the cutoff values of ESC Guidelines, hyperlipidemia was defined as elevated total cholesterol (TC), low-density lipoprotein cholesterol (LDL-C) or triglycerides (TG) or by the use of lipid-lowering medications (13). All patients were physically examined for heart rate, blood pressure, weight, height, and waist circumference (WC). Body mass index (BMI) was calculated according to the standard formula. Patients with proven ischemic heart disease or stroke, cardiomyopathy, type 1 diabetes, chronic renal disease stage IV or more, known thyroid gland disease, and active cancer were excluded from the study.

The study was approved by the local Ethical Committee at the Medical University of Varna (Protocol No 75/ 07.06.2018). Written informed consent was obtained from all participants in the study.

\section{Coronary Artery Calcification Measurement}

All participants were examined on a multi-slice computed tomography in order to assess the presence of coronary artery calcification. CAC scans were performed on a Siemens Somatom Definition (Dual Source $2 \times 64$ ) CT scanner (Erlangen, Germany) using standardized imaging protocols and were interpreted by a single trained physician blinded to the clinical characteristics of the patients. Coronary artery calcification was defined as a lesion above 130 Hounsfield units, with an area equal to three pixels. CAC score (CACS) was calculated using the Agatston criteria (14). The presence of CAC was defined as an Agatston score $>0$ Agatston units (AU). According to the CACS results, the patients were classified into one of the following categories: $\mathrm{CACS}=0$ $\mathrm{AU}$ (no coronary calcium), CACS $>0 \mathrm{AU}$ (presence of coronary calcium).

\section{Biochemical Measures}

Data for routine biochemical parameters, lipid profile parameters (TG, TC, HDL cholesterol, LDL cholesterol) and C-reactive protein (CRP) were extracted from the medical documentation. Fasting venous blood was drawn at admittance in the clinic. Blood samples were centrifuged at $1600 \mathrm{xg}$ for 15 $\mathrm{min}$, and blood plasma and serum were separated and stored at $-80^{\circ} \mathrm{C}$ until analysis of specific laboratory parameters. A commercial ELISA kits based on specific competitive mono-antibodies were used for measuring the plasma levels of total GRP (Develop, China), circulating ucMGP (Cusabio, Wuhan, China), uncarboxylated (ucOC), and carboxylated osteocalcin (cOC) (BlueGene Biotech, Shanghai, China). The ratio between the concentrations of circulating ucOC and cOC (UCR) was calculated and used as an inverse indicator of vitamin $\mathrm{K}$ status. A lower OCR reflects good vitamin $\mathrm{K}$ status (15).

\section{Statistical Analysis}

Data are presented as mean \pm standard deviation, or number (n) and percentage. Statistical analysis was performed by GraphPad software version 7 (GraphPad Software, San Diego, CA USA) and SPSS v.23 (SPSS Inc., Chicago, IL, USA). Student's ttest was used to compare the means of two groups. Spearman correlation analysis was used for evaluation of the associations between the tested variables. Statistical significance was considered at $\mathrm{p}<0.05$.

\section{RESULTS}

Table 1 represents the baseline patients' characteristics. Significant differences between CVD and non-CVD patients were indicated for age, anthropometric measurements, $\mathrm{AH}$ duration, and smoking status. CACS for CVD group was three times higher compared to the non-CVD controls. Significantly higher values were indicated for serum glucose, creatinine, urea, and uric acid. More pronounced changes of the tested lipid parameters were found for LDL cholesterol.

We indicated a clear tendency for a decrease in circulating GRP levels for CVD patients vs. nonCVD controls (Fig. 1). Although non-significant, the same trend was preserved when the CVD participants were divided according to CVD pathologylower GRP values were found for the HF group than those for AF patients $(1.06 \pm 0.8$ vs. $1.29 \pm 1.02 \mathrm{ng} / \mathrm{mL})$.

For assessing the changes in circulating GRP to the presence or absence of arterial calcium deposits, we stratified the patients according to their CACS. The data reveal a significantly lower circulating GRP (with 23.4\%) in the group with coronary calcium $(\mathrm{CACS}>0)$ vs. patients without $\mathrm{CAC}(\mathrm{CACS}=0)$ (Fig. 2).

To assess the link between circulating GRP and the tested risk factors for atherosclerosis we per- 
Is Circulating Gla-Rich Protein Linked with Coronary Calcium and Cardiovascular Pathology in Patients with Atrial Fibrillation...

Table 1. Patients' baseline characteristics

\begin{tabular}{|c|c|c|c|}
\hline Parameter & $\begin{array}{c}\text { Control } \\
\text { Mean } \pm \text { SD }(n=41)\end{array}$ & $\begin{array}{c}\text { CVD Patients } \\
\text { Mean } \pm \text { SD }(n=40)\end{array}$ & $\mathbf{p}$ \\
\hline Age (years) & $53.39 \pm 9.81$ & $68.88 \pm 7.03$ & 0.001 \\
\hline Male gender (\%) & 39.0 & 32.5 & 0.352 \\
\hline BMI $\left(\mathrm{kg} / \mathrm{m}^{2}\right)$ & $27.65 \pm 5.66$ & $30.36 \pm 5.61$ & 0.039 \\
\hline $\mathrm{WC}(\mathrm{cm})$ & $92.00 \pm 16.02$ & $104.89 \pm 12.91$ & 0.001 \\
\hline AH duration (years) & $6.11 \pm 5.21$ & $12.05 \pm 8.02$ & 0.001 \\
\hline Smoking duration (years) & $17.92 \pm 8.69$ & $28.69 \pm 12.66$ & 0.013 \\
\hline Diabetes duration (years) & $5.50 \pm 3.54$ & $12.93 \pm 12.5$ & 0.209 \\
\hline CACS (AU) & $80.84 \pm 285.45$ & $353.17 \pm 560.66$ & 0.007 \\
\hline Glucose $(\mathrm{mmol} / \mathrm{L})$ & $5.81 \pm 0.82$ & $7.44 \pm 3.34$ & 0.004 \\
\hline Urea $(\mathrm{mmol} / \mathrm{L})$ & $5.83 \pm 0.87$ & $8.37 \pm 4.99$ & 0.050 \\
\hline Creatinine $(\mathrm{mmol} / \mathrm{L})$ & $68.05 \pm 13.50$ & $83.24 \pm 23.76$ & 0.001 \\
\hline Uric acid (mmol/L) & $318.28 \pm 111.36$ & $388.96 \pm 99.51$ & 0.009 \\
\hline Total cholesterol (mmol/L) & $5.25 \pm 1.01$ & $4.86 \pm 1.19$ & 0.126 \\
\hline Triglycerides (mmol/L) & $1.41 \pm 1.04$ & $1.47 \pm 0.87$ & 0.765 \\
\hline HDL cholesterol (mmol/L) & $1.39 \pm 0.41$ & $1.44 \pm 0.45$ & 0.575 \\
\hline LDL cholesterol (mmol/L) & $3.26 \pm 0.76$ & $2.79 \pm 0.92$ & 0.016 \\
\hline CRP (mg/L) & $0.61 \pm 0.459$ & $5.54 \pm 6.19$ & 0.282 \\
\hline GRP (ng/mL) & $1.11 \pm 0.78$ & $0.91 \pm 0.77$ & 0.080 \\
\hline ucMGP $(\mu \mathrm{g} / \mathrm{mL})$ & $4.42 \pm 3.37$ & $5.69 \pm 4.94$ & 0.057 \\
\hline
\end{tabular}

Abbreviations: BMI—body mass index; AH—arterial hypertension; CACS—coronary arterial calcium score; HDL_high-density lipoprotein; LDL_low-density lipoprotein; CRP_C-reactive protein; GRP_Gla-rich protein; ucMGP_uncarboxylated matrix Gla protein.

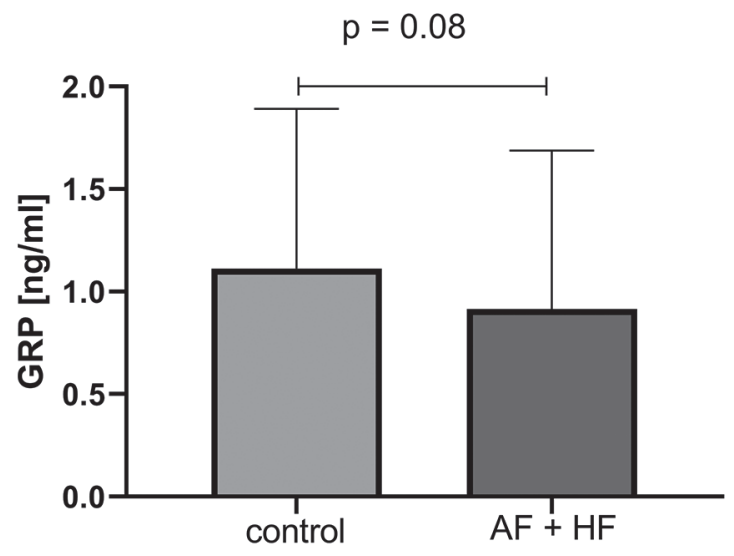

Fig. 1. Circulating serum GRP levels in controls (non-CVD patients) and in the combined CVD group (AF and HF patients).

Data are given as mean $\pm S D$; statistical significance was considered at $p<0.05$; GRP_Gla-rich protein; CVD_cardiovascular disease; $A F$-atrial fibrillation; $H F$ - heart failure with preserved ejection fraction.

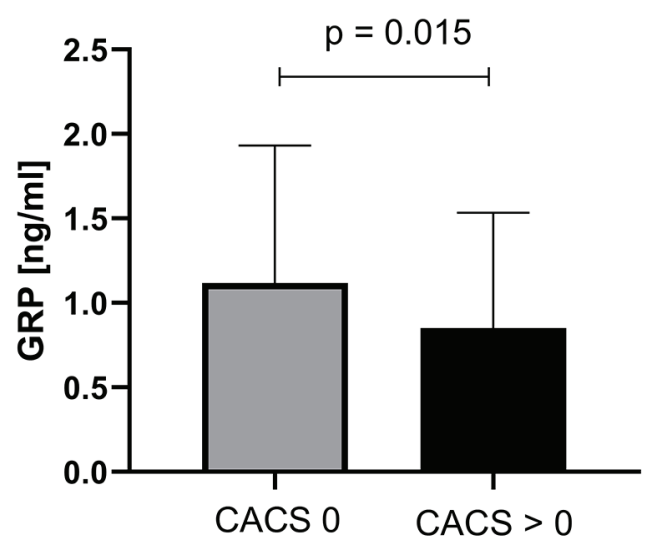

Fig. 2. Circulating serum GRP levels in patients without coronary calcium $(C A C S=0)$ and in participants with arterial calcium deposits (CACS $>0$ ).

Data are given as mean $\pm S D$; statistical significance was considered at $p<0.05$; GRP_Gla-rich protein; CACS - coronary arterial calcium score. 
Deyana Vankova, Milena Pasheva, Atanas Angelov et al.

formed Spearman correlation analysis for controls and CVD patients. Of the tested lipid parameters negative correlation with borderline significance was found for total cholesterol $(\mathrm{r}=-0.287 ; \mathrm{p}=0.056)$ and triglyceride levels $(\mathrm{r}=-0.293 ; \mathrm{p}=0.051)$ for the combined CVD group. For the same group a negative trend for a decrease in circulating GRP with CRP as a marker of inflammation ( $\mathrm{r}=-0.187 ; \mathrm{p}=0.416)$ was indicated. In the control non-CVD group we found significant negative associations only with smoking duration ( $r=-0.417 ; \mathrm{p}=0.0197)$. Serum GRP tended to decrease with CACS for CVD and non-CVD patients. Circulating GRP correlated positively with circulating ucMGP for the control subjects $(\mathrm{r}=0.288$; $\mathrm{p}=0.05)$ and for the combined CVD group $(\mathrm{r}=0.561$; $\mathrm{p}<0.0001$ ).

When the patients were divided according to their CACS, significant moderate association was found between circulating GRP and ucMGP and negative correlation with smoking status for both groups with or without coronary calcium. CRP, TC, TG and LDL-C tended to increase with the decrease of serum GRP (Table 2).

We did not found interrelations between circulating GRP, anthropometric parameters, duration of arterial hypertension, diabetes, both $\mathrm{cOC}$ and ucOC or OCR levels.

\section{DISCUSSION}

Our study aimed to estimate the relationship between circulating total GRP with CVD pathology and vascular calcification. In CVD patients (combined AF + HF group), we have also examined the interaction between GRP, certain risk factors for CVD and with the circulating levels of ucMGP as proven VC inhibitor.

We observed a tendency, although not significant, for a decrease in circulating levels of total GRP in the CVD patients, which is consistent with other studies $(6,16,17)$. In the study of Viegas et al. (7), the levels of serum GRP sharply decrease in chronic kidney disease (CKD) patients with the severity of CKD pathology, e.g. from stage $2 \mathrm{CKD}$ to stage $4 \mathrm{CKD}$. Our data also revealed lower serum GRP not only for the combined CVD group, but also for patients with more severe CVD pathology, such as heart failure.

Vascular calcification is a process under strict regulation of calcium and phosphate deposition in the intima and media layers of the vessel wall and in cardiac valves. The inhibition of mineral crystal formation is critical for prevention the destructive effects of calcium deposits and ectopic calcification. This process is actively inhibited by the functional calcification inhibitors, including MGP and fetuinA (18). GRP is another constitutive component of the inhibitory system composed of MGP and fetuinA preventing crystal formation and maturation (7). It is assumed that deficiency in GRP may result in increased mineral maturation promoting VC (7). In agreement with these data our study revealed significantly lower GRP levels in patients with coronary artery calcium $(\mathrm{CACS}>0)$ compared to those without coronary calcium $(\mathrm{CACS}=0)$.

In the present study, we found a positive association between circulating GRP and ucMGP in both CVD patients and in those with coronary calcium. To our knowledge, this is the first study examining the relation between these two inhibitory proteins of

Table 2. Spearman correlation analysis between circulating GRP, tested risk factors for CVD and biochemical parameters in patients with or without coronary calcium deposits. Statistical significance was considered at $p<0.05$.

\begin{tabular}{lc|c}
\hline \multirow{2}{*}{ Tested Parameter } & CACS $>0$ & CACS $=0$ \\
\cline { 2 - 3 } Smoking duration (years) & Spearman $\mathrm{r} ; \mathrm{p}$ & Spearman $\mathrm{r} ; \mathrm{p}$ \\
TC $(\mathrm{mmol} / \mathrm{L})$ & $-0.389 ; \mathrm{p}=0.045$ & $-0.386 ; \mathrm{p}=0.084$ \\
TG $(\mathrm{mmol} / \mathrm{L})$ & $-0.189 ; \mathrm{p}=0.193$ & $-0.048 ; \mathrm{p}=0.764$ \\
LDL-C $(\mathrm{mmol} / \mathrm{L})$ & $-0.148 ; \mathrm{p}=0.309$ & $-0.108 ; \mathrm{p}=0.496$ \\
CRP $(\mathrm{mg} / \mathrm{L})$ & $-0.155 ; \mathrm{p}=0.293$ & $-0.054 ; \mathrm{p}=0.733$ \\
$\mathrm{ucMGP}(\mu \mathrm{g} / \mathrm{mL})$ & $-0.044 ; \mathrm{p}=0.871$ & $-0.500 ; \mathrm{p}=0.267$ \\
\hline
\end{tabular}

Abbreviations: TC—-total cholesterol; TG-triglycerides; LDL-C-low-density lipoprotein cholesterol; CRP-C-reactive protein; ucMGP_undercarboxylated matrix Gla protein 
VC in the serum of CVD patients. It is not surprising that such positive relationship between circulating GRP and ucMGP as GRP is a component of a protein inhibitory complex (GRP-MGP-fetuin A) present at the sites of aortic valve calcification (7).

Our results demonstrate a clear trend for a decrease of total serum GRP with the increase of serum CRP for both CVD patients and in those with CACS $>0$. In addition, serum LDL levels also tend to increase with the decrease of circulating GRP. It is well known that calcification and inflammation are interconnected processes contributing to atherosclerosis development. There are data showing that decreased GRP levels are linked with increased pro-inflammatory responses of articular cells, and thus confirming the dual role of GRP - as calcification inhibitor and anti-inflammatory agent $(19,20)$. Thus, it is considered that GRP may have strong implications in calcification and/or inflammation-related diseases such as CVD (6).

One of the limitations of the current study is the small number of studied patients. The GRP assay used for measuring circulating GRP cannot distinguish carboxylated and uncarboxylated GRP forms. Our study, for the first time, demonstrates a decrease in circulating total GRP in patients with CVD and in those with coronary calcium deposits. We have shown a significant relationship between circulating GRP and ucMGP confirming the notion that these two Gla proteins are components of a complex VC inhibitory system. An association between circulating GRP and serum CRP and LDL levels was demonstrated, further contributing to the hypothesis regarding the dual role of GRP as a calcification inhibitor and an anti-inflammatory agent. Our findings add new information regarding the role of circulating GRP as a new player in calcification inhibition.

\section{CONCLUSION}

Our study illuminates the link between total circulating GRP and CVD pathology and the degree of coronary calcification. However, further research is needed to fully elucidate the molecular mechanisms of GRP action and to clarify its role as a potential novel valuable biomarker evaluating the development and the degree of cardiovascular risk and VC.

\section{Disclosure Statement:}

The authors report no potential conflict of interest.

\section{Funding:}

This work was supported by the Science Fund at the Medical University of Varna, Bulgaria under Grant Number 98/19.12.19.1.

\section{REFERENCES}

1. Roumeliotis S, Dounousi A, Salmas M, Elefteriadis T, Liakopoulos V. Vascular calcification in chronic kidney disease: the role of vitamin K- dependent matrix Gla protein. Front Med. 2020;7:154. doi: 10.3389/fmed.2020.00154.

2. Viegas C, Araújo N, Marreiros C, Simes D. The interplay between mineral metabolism, vascular calcification and inflammation in Chronic Kidney Disease (CKD): Challenging old concepts with new facts. Aging, 2019;11(12):4274-99. doi: 10.18632/ aging.102046.

3. Nelson AJ, Raggi P, Wolf M, Gold A, Chertow G, Roe MT. Targeting vascular calcification in chronic kidney disease. J Am Coll Cardiol Basic Transl Science. 2020; 5(4):398-412. doi: 10.1016/j. jacbts.2020.02.002.

4. Surmann-Schmitt C, Dietz U, Kireva T, Adam N, Park J, Tagariello A, et al. Ucma, a novel secreted cartilage-specific protein with implications in osteogenesis. J Biol Chem. 2008;283(11):7082-93. doi: 10.1074/jbc.M702792200.

5. Viegas CS, Simes DC, Laizé V, Williamson MK, Price PA, Cancela ML. Gla-rich protein (GRP), a new vitamin K-dependent protein identified from sturgeon cartilage and highly conserved in vertebrates. J Biol Chem. 2008;283(52):36655-64. doi: 10.1074/jbc.M802761200.

6. Viegas CS, Rafael MS, Enriquez JL, Teixeira A, Vitorino R, Luís IM, et al. Gla-rich protein acts as a calcification inhibitor in the human cardiovascular system. Arterioscler Thromb Vasc Biol. 2015;35(2):399-408. doi: 10.1161/ ATVBAHA.114.304823.

7. Viegas CS, Santos L, Macedo AL, Matos AA, Silva, AP, Neves PL, et al. Chronic kidney disease circulating calciprotein particles and extracellular vesicles promote vascular calcification: A role for GRP (Gla-rich protein). Arterioscler 
Deyana Vankova, Milena Pasheva, Atanas Angelov et al.

Thromb Vasc Biol. 2018; 38(3):575-87. doi: 10.1161/ ATVBAHA.117.310578.

8. Cavaco S, Viegas CS, Rafael MS, Ramos A, Magalhães J, Blanco FJ, et al. Gla-rich protein is involved in the cross-talk between calcification and inflammation in osteoarthritis. Cell Mol Life Sci. 2015;73(5):1051-65. doi: 10.1007/s00018-015-2033-9.

9. Willems BA, Vermeer C, Reutelingsperger CP, Schurgers $\mathrm{LJ}$. The realm of vitamin $\mathrm{K}$ dependent proteins: shifting from coagulation toward calcification. Mol Nutr Food Res. 2014;58(8):1620-35. doi: 10.1002/mnfr.201300743.

10. Luo G, Ducy P, Mckee MD, Pinero GJ, Loyer E, Behringer RR, et al. Spontaneous calcification of arteries and cartilage in mice lacking matrix Gla protein. Nature. 1997;386(6620):78-81. doi: 10.1038/386078a0.

11. Viegas CS, Herfs M, Rafael MS, Enriquez JL, Teixeira A, Luís IM, et al. Gla-rich protein is a potential new vitamin $\mathrm{K}$ target in cancer: evidences for a direct GRP-mineral interaction. Biomed Res Int. 2014;2014:340216. doi: 10.1155/2014/340216.

12. Rafael MS, Cavaco S, Viegas CS, Santos $S$, Ramos A, Willems B, et al. Insights into the association of Gla-rich protein (GRP) and osteoarthritis: novel splice variants and $\gamma$-carboxylation status. Mol Nutr Food Res. 2014;58(8):1636-46. doi: 10.1002/ mnfr.201300941.

13. Piepoli MF, Hoes A, Agewall S, Albus C, Brotons C, Catapano A, et al. European Guidelines on cardiovascular disease prevention in clinical practice: The sixth joint task force of the European society of cardiology and other societies on cardiovascular disease prevention in clinical practice (constituted by representatives of 10 societies and by invited experts). Developed with the special contribution of the European association for cardiovascular prevention \& Rehabilitation (EACPR). Eur Heart J.2016; 37(29):2315-81. doi: 10.1093/eurheartj/ ehw106.
14. Agatston AS, Janowitz WR, Hildner FJ, Zusmer NR, Viamonte M Jr, Detrano R. Quantification of coronary artery calcium using ultrafast computed tomography. J Am Coll Cardiol. 1990;15(4):827-32. doi: 10.1016/0735-1097(90)90282-t.

15. Dalmeijer GW, van der Schouw Y, Magdeleyns E, Vermeer C, Elias S, Velthuiset B, et al. Circulating species of matrix Gla protein and the risk of vascular calcification in healthy women. Int J Cardiol. 2013;168(6):e168-70. doi: 10.1016/j. ijcard.2013.08.062.

16. Willems BA, Furmanik M, Caron MM, Chatrou ML, Kusters DH, Welting TJ, et al. Ucma/GRP inhibits phosphate-induced vascular smooth muscle cell calcification via SMAD-dependent BMP signaling. Sci Rep. 2018;8(1):4961. doi: 10.1038/ s41598-018-23353-y.

17. Silva AP, Viegas CS, Mendes F, Macedo A, Guilherme P, Tavares N. Gla-Rich protein (GRP) as an early and novel marker of vascular calcification and kidney dysfunction in diabetic patients with CKD: A pilot cross-sectional study. J Clin Med. 2020;9(3):635. doi: 10.3390/jcm9030635.

18. Shroff R, Long DA, Shanahan C. Mechanistic insights into vascular calcification in CKD. J Am Soc Nephrol. 2013;24(2):179-89. doi: 10.1681/ ASN.2011121191.

19. Viegas CS, Cavaco S, Neves PL, Ferreira A, João A, Williamson MK, et al. Gla-rich protein is a novel vitamin K-dependent protein present in serum that accumulates at sites of pathological calcifications. Am J Pathol. 2009;175(6):2288-98. doi: 10.2353/ ajpath.2009.090474.

20. Ueland T, Gullestad L, Dahl CP, Aukrust P, Aakhus S, Solberg OG, et al. Undercarboxylated matrix Gla protein is associated with indices of heart failure and mortality in symptomatic aortic stenosis. J Intern Med. 2010;268(5):483-92. doi: 10.1111/j.1365-2796.2010.02264.x. 